\title{
Tamoxifen induces hepatotoxicity and changes to hepatocyte morphology at the early stage of endocrinotherapy in mice
}

\author{
FANG-FANG GAO ${ }^{1 *}$, JIA-WEI LV $^{2 *}$, YING WANG $^{1}$, RONG FAN $^{4}$, QUN LI $^{3}$, ZUN ZHANG $^{1}$ and LEI WEI ${ }^{1}$ \\ ${ }^{1}$ Department of Pathology and Pathophysiology, Wuhan University School of Basic Medical Sciences; ${ }^{2}$ Zhongnan Hospital \\ of Wuhan University, The Second College of Clinical Medicine of Wuhan University, Wuhan, Hubei 430071; ${ }^{3}$ Renmin Hospital \\ of Wuhan University, The First College of Clinical Medicine of Wuhan University, Wuhan, Hubei 430060; ${ }^{4}$ Department \\ of Physiology, Wuhan University School of Basic Medical Sciences, Wuhan, Hubei 430071, P.R. China
}

Received August 11, 2015; Accepted September 18, 2015

DOI: $10.3892 /$ br.2015.536

\begin{abstract}
Clinically, hepatotoxicity is an inevitable side effect during long-term endocrinotherapy in breast cancer patients. Various studies have reported the specific mechanism and protective methods for this long-term hepatotoxicity, however, the short-term influences of tamoxifen (TAM) on hepatocytes remain to be elucidated. The previous study investigated TAM-induced liver injury at the early stage of endocrine treatment. Mice were assigned into 2 groups: The experiment group was administrated with intraperitoneal (i.p.) injection of $6 \mathrm{mg} / \mathrm{kg} / \mathrm{day}$ TAM for 2 weeks, and the control group was administrated with i.p. injection of physiological saline of the same dose. Body weights in each group were detected every day, and alanine aminotransferase and aspartate aminotransferase levels were measured every 3 days. Small pieces of the liver tissues were obtained and processed for protein extraction, biochemical detection and histopathological analysis 2 weeks later. The results indicated that TAM decreased the mice body weights. Morphologically, with the treatment of TAM for only 2 weeks, at the microscopic and ultrastructural levels the structure of hepatic cords became blurred in sections of the regions, although the lobules of the liver remained visible. Partially, hepatic cells were swelled in spherical shapes. Nuclei appeared to be pyknotic and exhibited uneven chromatin distribution. In addition, it was observed in the transmission electron microscopy analysis that nuclei became pyknotic and unevenly distributed. The majority of the nuclei were endowed with distinct heterochromatin and thick nucleoli. The mitochondrial cristae became vague and disorganized. Finally, western blotting was used and detected a significant increase of the caspase-3 level in the liver
\end{abstract}

Correspondence to: Professor Lei Wei, Department of Pathology and Pathophysiology, Wuhan University School of Basic Medical Sciences, 185 Dong Hu Road, Wuhan, Hubei 430071, P.R. China E-mail: leiweifr@hotmail.com

*Contributed equally

Key words: tamoxifen, hepatotoxicity, short-term treatment, breast cancer tissues. In conclusion, the experiments elucidated that TAM ( $6 \mathrm{mg} / \mathrm{kg} / \mathrm{day}$ ) would cause hepatotoxicity at the early stage of endocrine treatment in mice, and the underlying mechanism was involved with hepatocyte apoptosis.

\section{Introduction}

Breast cancer is one of the most common carcinoma risks worldwide, particularly females (1). According to an overview that referred to the female breast cancer statistics for 2013 in the United States (US), it was reported that 232,340 new cases of invasive breast cancer and 39,620 breast cancer fatalities were predicted among US women in 2013. It has been estimated that 1 in 8 women in the US will develop breast cancer in their lifetimes (2). In developing countries including China, breast cancer ranks only second to cervical cancer in female morbidity and mortality (3).

According to the epidemiological investigation, nearly $70 \%$ of patients with breast carcinomas are eligible for endocrine adjuvant treatment due to the expression of the estrogen receptor- $\alpha(E R \alpha)$ (4). Selective ER modulators (SERMs) are synthetic non-steroidal compounds that switch target sites on and off throughout the body. Tamoxifen (TAM), the pioneering SERM, which acts to block estrogen action by binding to the ER in breast carcinomas, has been used ubiquitously in clinical treatment during the last 30 years and is proved to reduce the risk of breast cancer in high-risk women with numerous clinical evidence $(5,6)$.

Although viewed as highly effective in anticancer endocrinotherapy, the various side effects must be considered. The recent studies have already reported the numerous long-term side effects, as patients are recommended to continue the treatment for $>5$ years. The long-term use of TAM may cause ranges of side effects such as hot flashes, night sweats, gynecological symptoms (vaginal dryness and vaginal discharge), depression, memory loss, sleep alterations and diminished sexual function (7,8). Among all these, 3 of the most serious adverse events are endometrial hyperplasia or endometrial cancer, venous thromboembolic disease, and hepatic injury or even hepatocarcinoma (which has only been reported in rats) (9-11). Recently, there were numerous studies that illustrated the specific mechanism of TAM-induced hepatotoxicity, however, studies on TAM-induced morphological 
changes in hepatic injury remain to be elucidated, particularly in its early stage of long-term endocrinotherapy $(12,13)$.

In the present study, the hepatotoxicity effects of TAM treatment at the early stage of endocrinotherapy were elucidated. Additionally, the morphological changes of hepatocytes in TAM-treated mice were observed at the microscopic and ultrastructural levels.

\section{Materials and methods}

Reagents. TAM (Wuhan Wei Shunda Technology Co., Ltd., Wuhan, China), Radioimmunoprecipitation Assay (RIPA; Sigma, St. Louis, MO, USA) triturator, rabbit polyclonal anti-human caspase-3, $\beta$-actin, horseradish peroxidase (HRP)-linked goat anti-rabbit and HRP-linked goat anti-mouse (Santa Cruz Biotechnology, Inc., Dallas, TX, USA) were used.

Western blotting. Mouse liver tissues were lysed in modified RIPA buffer. The protein concentration was measured by the $\mathrm{BCA}^{\mathrm{TM}}$ protein assay kit (Pierce Biotechnology, Inc., Rockford, IL, USA). Equal amounts of total protein $(20 \mu \mathrm{g})$ were loaded and run on a $5 \%(\mathrm{v} / \mathrm{v})$ sodium dodecyl sulphate (SDS)-polyacrylamide stacking gel and 10\% (v/v) SDS-polyacrylamide gradient gel, and were subsequently transferred to polyvinylidene fluoride membranes (Roche Carolina, Florence, SC, USA). Membranes were blocked for $1 \mathrm{~h}$ at room temperature with $5 \%$ powdered skimmed milk in Tris-buffered saline with $0.1 \%$ Tween-20, and were subsequently probed with caspase-3 antibody (cat. no. CB91021324; 1:1,000 dilution; Alexis Biochemicals, San Diego, CA, USA) or $\beta$-actin antibody (cat. .no. 874526765; 1:2,000 dilution; Roche Carolina) at $4^{\circ} \mathrm{C}$ overnight. After incubation with HRP-linked secondary antibodies (sc55227; 1:5,000, Santa Cruz Biotechnology, Inc.) for $2 \mathrm{~h}$ at room temperature, the blots were detected with and ECL kit (Easy Blot, Sangon Biotech, Shanghai, China). The experiments were repeated 3 times.

Animal experiment. Female inbred 7-8-week-old Kunming mice were purchased from the ABSL-III laboratory at Wuhan University (Wuhan, Hebei, China). All the mice were fed under identical conditions in an aseptic facility and provide free access to water and food. Mice were randomly allocated to two groups with 6 for each. Group I were labeled as the control group, administrated with intraperitoneal (i.p.) injection of physiological saline of the same dose for 2 weeks (the average weight of mice was $27 \mathrm{~g}$ ). Group II were labeled as the TAM group, administrated with i.p. injection of $6 \mathrm{mg} / \mathrm{kg} /$ day TAM for 2 weeks. The body weight of each mouse was detected to evaluate the effects of the treatment. At the end of the time period, mice were euthanized by carbon dioxide. Immediately following the sacrifice of the animals, small pieces of liver tissues were obtained and processed for histopathological analysis and transmission electron microscopy (TEM) examination. All the animal work was performed in accordance with protocols and guidelines approved by the Institutional Animal Care and Use Committee (Wuhan University).

Determination of the serum biochemical parameters. Activities of enzyme markers of hepatocellular damage, including alanine aminotransferase (ALT) and aspartate aminotransferase (AST) in serum were determined every three days. All the analyses were performed in triplicate for every sample using a Semi-automatic Biochemical Analyzer (Vital Scientific, Spankeren, Netherlands).

Hepatic cell growth and morphology under an inverted microscope. Liver tissue sections were collected and fixed in $4 \%$ paraformaldehyde in $0.1 \mathrm{M}$ of phosphate-buffered saline (PBS) at room temperature overnight. Paraffin-embedded tissue sections $(\leq 5 \mu \mathrm{m})$ were stained with hematoxylin and eosin, according to standard techniques. Images were captured using a Motic BA200 microscope (Motic Instruments, Inc., Baltimore, MD, USA).

Ultrastructural changes under TEM. Liver tissues were cut into $1-\mathrm{mm}^{3}$ fragments and fixed by immersion in $4 \%$ prechilled glutaraldehyde in PBS [0.1 M (pH 7.4)] overnight at $4^{\circ} \mathrm{C}$. Following this, the samples were washed in PBS four times followed by post-fixation with $1 \%$ osmium tetroxide in $0.1 \mathrm{M}$ PBS for $2 \mathrm{~h}$ at $4^{\circ} \mathrm{C}$. Samples were dehydrated in graded ethanol, embedded in Epon 812, and subsequently cut into ultra- or semi-thin sections. The sections were examined under a transmission electron microscope (Hitachi HT7700-SS; Hitachi, Tokyo, Japan).

Statistical analysis. All the assays were performed at least in triplicate and data are expressed as mean \pm standard error (SE). The SE was calculated by dividing the standard deviation by the square root of the number of observations. Paired t-test was carried out to compare populations using GraphPad Prism software (GraphPad Software, La Jolla, CA, USA). P <0.05 was considered to indicate a statistically significant difference.

\section{Results}

Effects of TAM on body weight. At the beginning of the treatment, the weight of each mouse was detected, which was in the range of 27-30 g. Subsequently, the experimental group was injected with $6 \mathrm{mg} / \mathrm{kg} /$ day TAM for 2 weeks, while the vehicle control group was injected with the same volume of physiological saline. The body weights were measured every day, as shown in Fig. 1. The results indicated that the average weight in the TAM-treated group began to decrease significantly on the fourth day, compared with the control group in which body weights increased slightly and the difference was significant $(\mathrm{P}<0.01)$. The average body weight of the experimental group decreased by $32.7 \%$ in total following the treatment of TAM compared with the vehicle control.

Effects of TAM on serum biochemical parameters. As mice in the experimental group were administrated with i.p. injection of $6 \mathrm{mg} / \mathrm{kg} /$ day TAM, AST and ALT concentrations in the serum were detected every 3 days, in order to evaluate the effects of TAM on the metabolic function of the liver. As shown in Fig. 2, the ALT level began to increase from the third day compared with the control group and the results were significant $(\mathrm{P}<0.05)$. The AST level began to increase from day 6 and the results were significant $(\mathrm{P}<0.01)$. At day 15 , the total levels of AST and ALT activities were elevated by 60.8 and $125 \%$, respectively in the TAM-treated group compared to the saline control group. This occurred mainly due to leakage of these 


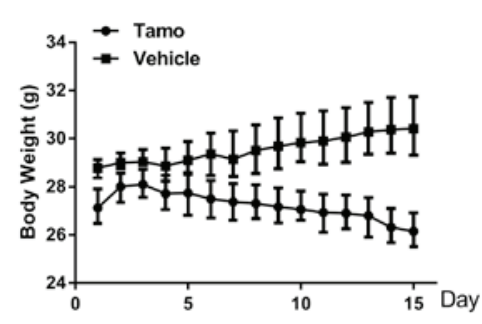

Figure 1. Effects of tamoxifen (TAM) on body weight. The average weight in the TAM-treated group began to decrease significantly on day 4 compared with the control group $(\mathrm{P}<0.01)$. Three independent experiments were performed. Results are presented as mean \pm standard deviation. $\mathrm{n}=6$ for each group.

$\mathbf{A}$

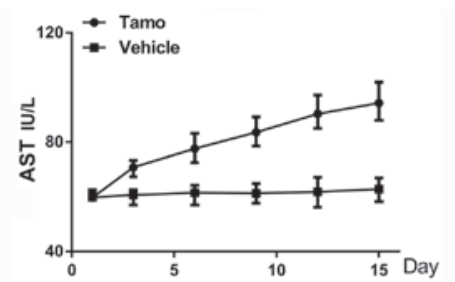

B

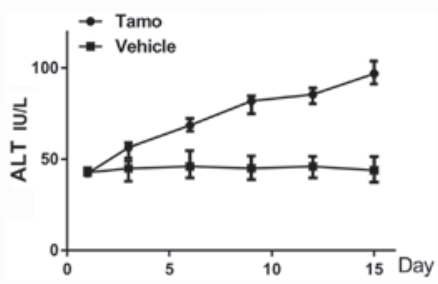

Figure 2. Aspartate aminotransferase (AST) and alanine aminotransferase (ALT) concentrations in serum following tamoxifen (TAM) treatment (A) During the 2-week administration of tamoxifen, the ALT level began to increase significantly from day 3 after the treatment, and 2 weeks later the total ALT activity level was elevated by $125 \%$ in the TAM-treated group compared to the saline control group $(\mathrm{P}<0.05)$. (B) The AST level began to increase significantly from day 6 after the treatment and 2 weeks later the total AST activity level was elevated by $60.8 \%$ in the TAM-treated group compared to the saline control group $(\mathrm{P}<0.01)$. Three independent experiments were performed. Results are presented as mean \pm standard deviation.

enzymes from damaged hepatocytes into the bloodstream, which manifested that a low dose of TAM treatment would lead to the damage of hepatocytes 2 weeks after the injection.

Morphological changes of liver tissue under an inverted light microscope. After being treated with $6 \mathrm{mg} / \mathrm{kg} /$ day TAM for 2 weeks, small pieces of liver tissue were obtained and processed for histopathological analysis. Fig. 3A and B represented the normal structure in the control group. Fig. 3C and D demonstrated that liver injury was caused by TAM. The structure of the hepatic lobules clearly became blurred in certain sections of the regions. Namely, the folial central vein expanded and engorged. Additionally, hepatic cells in certain areas swelled into spherical shapes and fatty metamorphosis was observed fractionally. Nuclei appeared to be pyknotic and exhibited uneven chromatin distribution. Furthermore, the cholestasis was also visible.

Liver cell ultrastructural changes under TEM. To further explore the ultrastructural changes of the hepatic cells, TEM was applied to examine its morphological changes in the TAM-treated group. As shown in Fig. 4, in the control group,
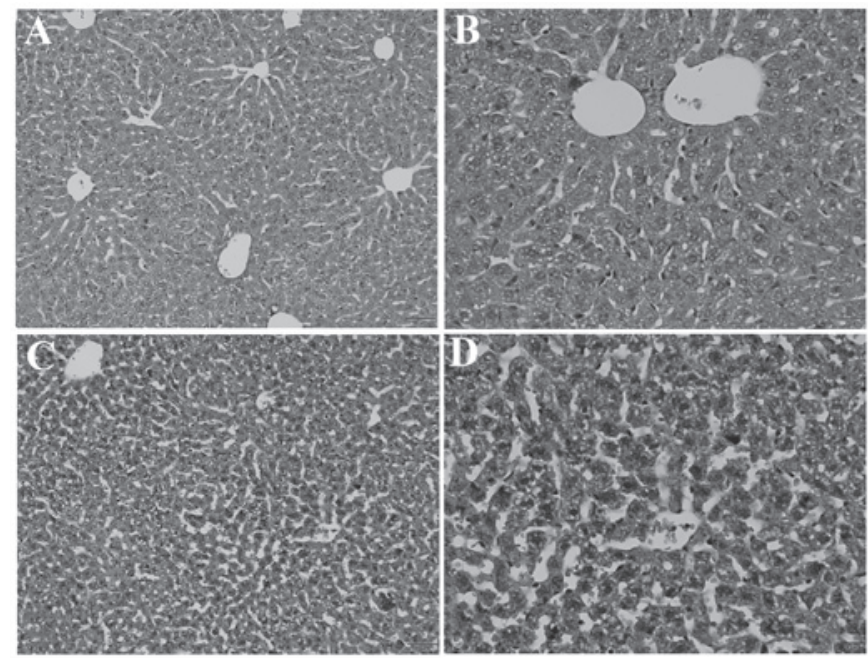

Figure 3. Morphological changes under electron microscopy. (A) The normal liver tissue structure in the control group (original magnification, x160). (B) The normal liver tissue structure in the control group (original magnification, $\mathrm{x} 400)$. (C and D) The liver tissue structure in the tamoxifen-treated group after the administration of tamoxifen for 2 weeks [original magnification, (C) x160 and (D) x400].

normal hepatocytes appeared to be polygonal in outline, and the central area had a large nucleus and clear mitochondria (Fig. 4A). However, the nuclei were pyknotic and unevenly distributed in the TAM-treated group. The majority of the nuclei exhibited distinct heterochromatin and thick nucleoli. The chromatins became coarse, granular and agglutinated in the nuclei (Fig. 4B and C). Hepatocytes appeared to have undergone vacuolar degeneration with lipid droplets diffusely distributed in the cytoplasm. In addition, mitochondrial cristae became vague and disorganized (Fig. 4D). Furthermore, the cholestasis was also visible under electron microscopy. (Fig. 4E and F).

Effects of TAM on caspase-3 expression level in liver tissues. To further illustrate the specific mechanisms that were involved in TAM-induced hepatotoxicity, the western blot assay was used to detect the caspase- 3 expression level in the liver tissues. $\beta$-actin served as a loading control. Representative blots from three independent experiments with similar results are shown in Fig. 5. The caspase- 3 expression level increased in the TAM-treated group compared with the vehicle control, and the difference was statistically significant $(\mathrm{P}<0.01)$. As it is well-recognized that caspase- 3 is closely involved in cell apoptosis, these results implicated that one of the mechanisms involved in TAM-induced hepatotoxicity was hepatocyte apoptosis.

\section{Discussion}

Worldwide, breast cancer is one of the most prevailing carcinomas in females. In America, breast cancer is the most common malignancy in females, which accounts for $>40,000$ fatalities each year (14). Clinically, based on gene expression profiling [ER, progesterone receptor (PR) and human epidermal growth factor receptor-2 (HER-2)], breast cancer has been classified into 4 major subtypes: Luminal A (ER/PR ${ }^{+}$, HER-2-), luminal B (ER/PR $\left.{ }^{+}, \mathrm{HER}-2^{+}\right)$, HER-2 overexpressing (ER/PR - HER-2 $\left.{ }^{+}\right)$and basal-like (ER/PR', 

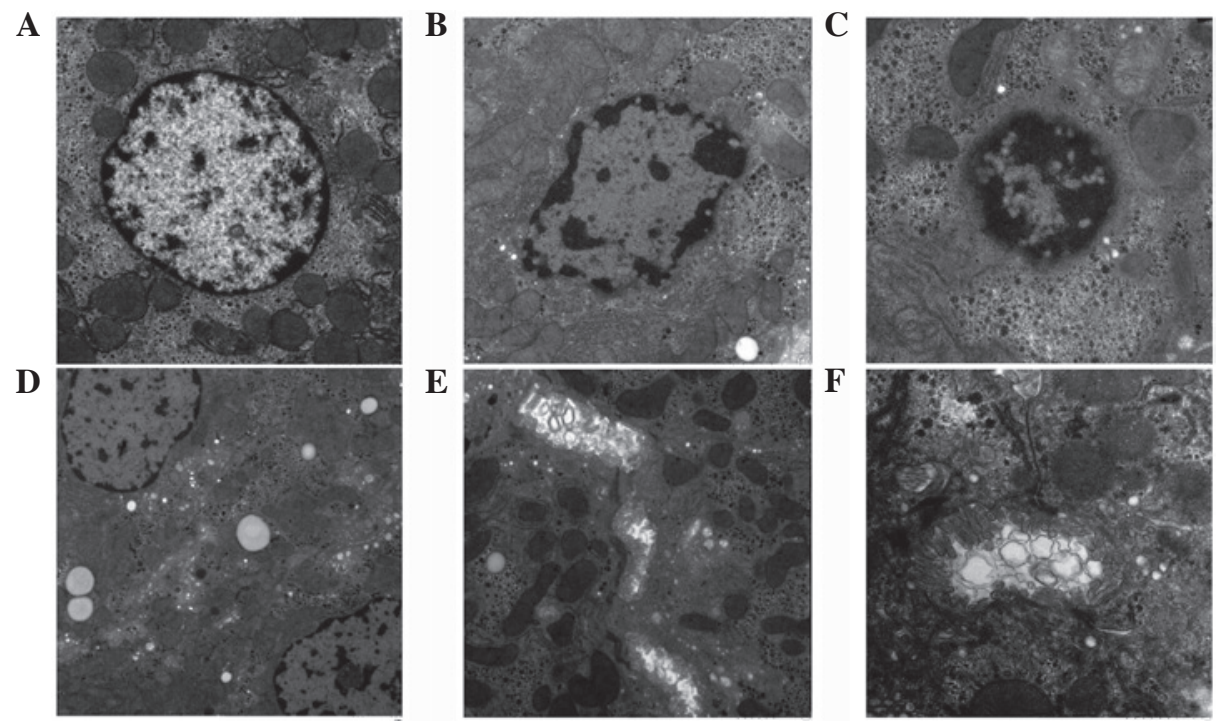

Figure 4. Ultrastructural changes under transmission electronmicroscopy. (A) The normal liver tissue structure in the control group (original magnification, $\mathrm{x} 3,000$ ). (B) After the administration of tamoxifen for two weeks, the liver tissue structure in the tamoxifen-treated group (original magnification, $\mathrm{x} 4,000$ ). (C-F) The liver tissue structure in the tamoxifen-treated group [original magnification, (C) x5,000, (D) x2,000, (E) x3,000 and (F) x7,000].
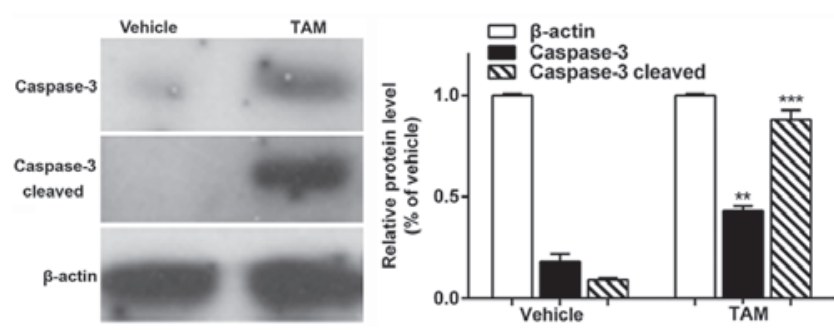

Figure 5. Caspase-3 expression level in the liver tissues. After the administration of tamoxifen (TAM) for 2 weeks, the caspase-3 expression level increased in the TAM-treated mice liver tissue compared with the vehicle control, and the difference was statistically significant $(\mathrm{P}<0.01)$. $\beta$-actin served as a loading control. Representative blots from three independent experiments with similar results. ${ }^{* *} \mathrm{P}<0.01,{ }^{* * *} \mathrm{P}<0.001$.

HER-2-) (15,16). For the two-thirds of high-risk breast cancer patients that are positive for ER and/or PR, endocrine therapy with TAM or aromatase inhibitors is generally recommended as highly effective $(17,18)$. The previous studies also provided significant evidence that 5-year standard TAM therapy would improve their 5-year survival rate, particularly in postmenopausal women (19). Additionally, the initial results from the first International Breast Cancer Intervention Study-I revealed that prophylactic use of TAM reduced the risk of invasive ER-positive tumors by $31 \%$ in women who were at an increased risk for breast cancer (20).

However, recurrences and side effects have restricted its long-term use to a large extent. Recent studies have revealed that long-term used of TAM may cause ranges of side effects such as hot flashes, night sweats, gynecological symptoms (vaginal dryness and vaginal discharge), depression, memory loss, sleep alterations, weight gain and diminished sexual function (7). Among all these, hepatic injury or even hepatocarcinoma would be one of the most severe side effects, which hindered its long-term use (9-11). Clinically, patients who accept the endocrinotherapy are instructed to reexamine their liver function every 4 months due to its hepatotoxicity. Numerous research and clinical studies have illustrated clearly that TAM causes the inhibition of mitochondrial $\beta$-oxidation and subsequently leads to macrovacuolar steatosis $(21,22)$. The early symptoms were characterized by the presence of a single, large lipid vacuole within the cytoplasm of the hepatocytes (23).

However, Carthew et al (24) reported that treatment of Wistar-AP female rats with dietary TAM for only 3 months was sufficient to cause cumulative hepatic DNA damage, which would finally develop into hepatocarcinoma with or without the promotion of phenobarbital. In addition, White et al (25) also elucidated that following the administration of TAM for 7 days (45 mg/kg/day) and extraction of hepatic DNA, $\leq 7$ radiolabelled adduct spots could be detected, as it caused a time-dependent increase in the level of adduct detected, up to a value of $\geq 1$ adduct $/ 10^{6}$ nucleotides after 7 days of dosing. These short-term emerged TAM genotoxicity results instigated the exploration of whether short-term TAM treatment would cause visible hepatotoxicity at the morphological level or whether a low dose of TAM would lead to hepatic injury.

Thus, the present study aimed to investigate whether TAM at a relatively low dose influences liver function at the early stage of the treatment. At the animal level, $6 \mathrm{mg} / \mathrm{kg} /$ day TAM was selected for 2-week treatment to build a short-term animal model, while the normal clinical dose for patients is $0.33 \mathrm{mg} / \mathrm{kg} /$ day (20 mg/day) and the scientific research dose for mice is $25 \mathrm{mg} / \mathrm{kg} /$ day $(26,27)$. During the process, mice body weights were detected every day and the AST and ALT levels in serum were measured every three days. The results revealed that TAM exerted distinct reducing effects on body weight in the 2-week treatment. In addition, the levels of ALT and AST in liver tissues were increased significantly when compared with the control group, and this is known to occur mainly due to the leakage of these enzymes from the damaged hepatocytes into the bloodstream. Subsequently, the morphological changes of hepatocytes were observed in the TAM-treated group, which changed evidently at the microscopic and ultrastructural levels. The structure of the hepatic lobules became blurred in sections 
of the region. Namely, the folial central vein expanded and engorged. Additionally, hepatic cells in certain sections of the regions swelled to spherical shapes and fatty metamorphosis was observed fractionally. Furthermore, cholestasis was also visible in certain areas. Under the TEM analysis, it was observed that the nuclei were pyknotic and unevenly distributed in TAM-treated group. The majority of the nuclei were endowed with distinct heterochromatin and thick nucleoli. The chromatins became coarse, granular and agglutinated in the nuclei. Hepatocytes appeared to undergo vacuolar degeneration with lipid droplets diffusely distributed in the cytoplasm. In addition, mitochondrial cristae became vague and disorganized. Finally, the specific mechanism that was involved in this hepatotoxicity was explored. A recent study elucidated that cell apoptosis has an important role in the process of hepatic cell injury (28). Thus, western blotting was applied to detect the expression level of caspase-3 in liver tissues, which had already been proved as one of the core proteins that participated in cell apoptosis. The results indicated that the caspase-3 expression level increased significantly in the liver tissues compared with the control group. This indicated that apoptosis had a vital role in this TAM-induced liver injury.

In conclusion, the present data showed that a relatively low concentration of TAM $(6 \mathrm{mg} / \mathrm{kg} /$ day $)$ for a short time treatment ( 2 weeks) would cause hepatotoxicity and change morphology at the microscopic and ultrastructural levels. Although the liver function may compensate or reverse the injuries gradually, the damage that occurred in the short-term TAM therapy has been shown. Thus, there is a necessity to obtain measures for monitoring liver function and protection at the early stage of the TAM endocrinotherapy, prior to apparent and undesirable clinical symptoms occurring. Furthermore, as DNA damage also occurs at this early period without clear clinical symptoms, which in the long-run increases the risk of hepatocarcinoma, exploring alternatives for TAM in long-term clinical endocrinotherapy is required.

\section{Acknowledgements}

The present study was supported by the Fundamental Research Funds for the Central Universities (grant no. 2014301020203).

\section{References}

1. Weycker D, Edelsberg J, Kartashov A, Barron R and Lyman G: Risk and healthcare costs of chemotherapy-induced neutropenic complications in women with metastatic breast cancer Chemotherapy 58: 8-18, 2012.

2. DeSantis C, Ma J, Bryan L and Jemal A: Breast cancer statistics, 2013. CA Cancer J Clin 64: 52-62, 2014.

3. Raftopoulou M and Hall A: Cell migration: Rho GTPases lead the way. Dev Biol 265: 23-32, 2004.

4. Hoppe R, Achinger-Kawecka J, Winter S, Fritz P, Lo WY, Schroth W and Brauch H: Increased expression of miR-126 and miR-10a predict prolonged relapse-free time of primary oestrogen receptor-positive breast cancer following tamoxifen treatment. Eur J Cancer 49: 3598-3608, 2013.

5. Jordan VC and O'Malley BW: Selective estrogen-receptor modulators and antihormonal resistance in breast cancer. J Clin Oncol 25: 5815-5824, 2007.

6. Moerkens M, Zhang Y, Wester L, van de Water B and Meerman JH: Epidermal growth factor receptor signalling in human breast cancer cells operates parallel to estrogen receptor $\alpha$ signalling and results in tamoxifen insensitive proliferation. BMC Cancer 14: 283, 2014.
7. Lorizio W, Wu AH, Beattie MS, Rugo H, Tchu S, Kerlikowske K and Ziv E: Clinical and biomarker predictors of side effects from tamoxifen. Breast Cancer Res Treat 132: 1107-1118, 2012.

8. Yeh WL, Lin HY, Wu HM and Chen DR: Combination treatment of tamoxifen with risperidone in breast cancer. PLoS One 9: e98805, 2014

9. Cuzick J, Powles T, Veronesi U, Forbes J, Edwards R, Ashley S and Boyle P: Overview of the main outcomes in breast-cancer prevention trials. Lancet 361: 296-300, 2003.

10. Hendrick A and Subramanian VP: Tamoxifen and thromboembolism. JAMA 243: 514-515, 1980.

11. Yang G, Nowsheen S, Aziz K and Georgakilas AG: Toxicity and adverse effects of Tamoxifen and other anti-estrogen drugs. Pharmacol Ther 139: 392-404, 2013.

12. El-Ashmawy NE and Khalil RM: A review on the role of L-carnitine in the management of tamoxifen side effects in treated women with breast cancer. Tumour Biol 35: 2845-2855, 2014.

13. Suddek GM: Protective role of thymoquinone against liver damage induced by tamoxifen in female rats. Can J Physiol Pharmacol 92: 640-644, 2014.

14. Al-Hajj M, Wicha MS, Benito-Hernandez A, Morrison SJ and Clarke MF: Prospective identification of tumorigenic breast cancer cells. Proc Natl Acad Sci USA 100: 3983-3988, 2003.

15. Perou CM, Sørlie T, Eisen MB, van de Rijn M, Jeffrey SS, Rees CA, Pollack JR, Ross DT, Johnsen H, Akslen LA, et al: Molecular portraits of human breast tumours. Nature 406: 747-752, 2000.

16. Goldhirsch A, Wood WC, Coates AS, Gelber RD, Thürlimann B and Senn HJ; Panel members: Strategies for subtypes - dealing with the diversity of breast cancer: Highlights of the St. Gallen International Expert Consensus on the Primary Therapy of Early Breast Cancer 2011. Ann Oncol 22: 1736-1747, 2011.

17. Howell A, Cuzick J, Baum M, Buzdar A, Dowsett M, Forbes JF, Hoctin-Boes G, Houghton J, Locker GY and Tobias JS; ATAC Trialists' Group: Results of the ATAC (Arimidex, Tamoxifen, Alone or in Combination) trial after completion of 5 years adjuvant treatment for breast cancer. Lancet 365: 60-62, 2005.

18. Mauri D, Pavlidis N, Polyzos NP and Ioannidis JP: Survival with aromatase inhibitors and inactivators versus standard hormonal therapy in advanced breast cancer: Meta-analysis. J Natl Cancer Inst 98: 1285-1291, 2006.

19. Tamoxifen for early breast cancer: An overview of the randomised trials. Early Breast Cancer Trialists' Collaborative Group. Lancet 351: 1451-1467, 1998.

20. Cuzick J, Forbes JF, Sestak I, Cawthorn S, Hamed H, Holli K and Howell A; International Breast Cancer Intervention Study I Investigators: Long-term results of tamoxifen prophylaxis for breast cancer - 96-month follow-up of the randomized IBIS-I trial. J Natl Cancer Inst 99: 272-282, 2007.

21. Lee MH, Kim JW, Kim JH, Kang KS, Kong G and Lee MO: Gene expression profiling of murine hepatic steatosis induced by tamoxifen. Toxicol Lett 199: 416-424, 2010.

22. Murata Y, Ogawa Y, Saibara T, Nishioka A, Fujiwara Y, Fukumoto M, Inomata T, Enzan H, Onishi S and Yoshida S: Unrecognized hepatic steatosis and non-alcoholic steatohepatitis in adjuvant tamoxifen for breast cancer patients. Oncol Rep 7: 1299-1304, 2000.

23. Labbe G, Pessayre D and Fromenty B: Drug-induced liver injury through mitochondrial dysfunction: Mechanisms and detection during preclinical safety studies. Fundam Clin Pharmacol 22: 335-353, 2008.

24. Carthew P, Martin EA, White IN, De Matteis F, Edwards RE, Dorman BM, Heydon RT and Smith LL: Tamoxifen induces short-term cumulative DNA damage and liver tumors in rats: Promotion by phenobarbital. Cancer Res 55: 544-547, 1995.

25. White IN, de Matteis F, Davies A, Smith LL, Crofton-Sleigh C, Venitt S, Hewer A and Phillips DH: Genotoxic potential of tamoxifen and analogues in female Fischer F344/n rats, DBA/2 and C57BL/6 mice and in human MCL-5 cells. Carcinogenesis 13: 2197-2203, 1992.

26. El-Beshbishy HA: Hepatoprotective effect of green tea (Camellia sinensis) extract against tamoxifen-induced liver injury in rats. J Biochem Mol Biol 38: 563-570, 2005.

27. El-Beshbishy HA: The effect of dimethyl dimethoxy biphenyl dicarboxylate (DDB) against tamoxifen-induced liver injury in rats: DDB use is curative or protective. J Biochem Mol Biol 38: 300-306, 2005.

28. Vince AR, Hayes MA, Jefferson BJ and Stalker MJ: Hepatic injury correlates with apoptosis, regeneration, and nitric oxide synthase expression in canine chronic liver disease. Vet Pathol 51: 932-945, 2014 